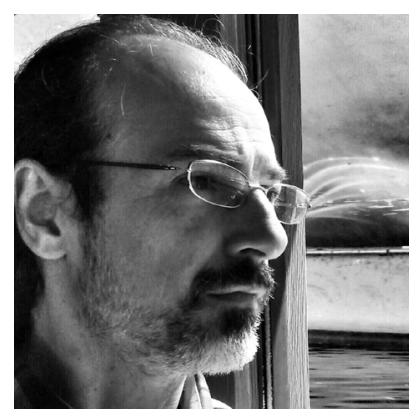

Diego Hurtado

Dr. en Física

Escuela de Humanidades

Universidad Nacional de San

Martin (UNSAM).

dhurtado@unsam.edu.ar

\title{
Proyectos de país en disputa: ¿Qué hacer con la ciencia y la tecnología?
}

Resumen: Frente al escenario electoral de octubre de 2019, se discuten las condiciones de posibilidad para que el sector de ciencia y tecnología (CyT) pueda asumir un papel político protagónico. Con este fin, se presenta una contextualización centrada en el lugar geopolítico de la Argentina y en la trayectoria de sus políticas de CyT. La oscilación pendular alrededor de proyectos de país en disputa y los aprendizajes del último período progresista (2003-2015) permiten inferir elementos de política para la recuperación perentoria, expansiva y enraizada de las actividades de CyT. Palabras clave: Semiperiferia, política de ciencia y tecnología, péndulo argentino, Estado

\section{National projects in dispute: what to do with science and technology?}

Abstract: Faced with the electoral scenario of October 2019, we discuss the conditions of possibility for the science and technology sector (S\&T) to assume a more central political political role. With this aim, a contextualization centered on the geopolitical place of Argentina and the trajectory of its S\&T policies is presented. The pendular oscillation between opposed National projects and lessons learned from the last progressive period (2003-2015) enables the statement of policy elements for the urgent, expansive and embedded recovery of S\&T activities.

Keywords: Semiperiphery, science and technology policy, Argentine pendulum, State

\section{Projetos de país em disputa: o que fazer com ciência e tecnologia?}

Resumo: Diante do cenário eleitoral de outubro de 2019, são discutidas as condições de possibilidade para que o setor de ciência e tecnologia (S\&T) assuma um papel político de destaque. Para tanto, apresenta-se uma contextualização centrada no lugar geopolítico da Argentina e na trajetória de suas políticas de S\&T. A oscilação pendular em torno de projetos de país em disputa e os aprendizados do último período progressivo (2003-2015) nos permitem inferir elementos de política para a recuperação definitiva, expansiva e enraizada das atividades de S\&T Palavras-chave: Semi-periferia, política de ciência e tecnologia, pêndulo 
| Ciencia, Tecnología y Política | Año 2 | N²2 | Enero-Junio 2019 | ISSN 2618-3188 | www.revistas.unlp.edu.ar/CTyP |

"Confundir la realidad europea, o norteamericana, con la realidad sin más es una falacia de desubiquidad (falacia de tomar el espacio o el mundo de otra cultura como el propio, encubriendo la originalidad distinta de la otra y la diferencia con la propia)"

\section{Enrique Dussel,}

Filosofías del Sur (2016), p. 92

\section{Introducción}

La recuperación en octubre de 2019 de un proyecto de país centrado en la producción, el trabajo y la inclusión representa una oportunidad histórica para que el sector de ciencia y tecnología (CyT) argentino pueda comenzar a concebirse a sí mismo como un actor político protagónico en la tarea de superar definitivamente el perfil de país atrapado en la telaraña geopolítica de una semiperiferia dependiente. Sin embargo, habría que preguntar primero si existen las condiciones para esta transformación. Y, si así fuera, ¿qué rasgos culturales habría que modificar (superar), qué tipo de capacidades organizacionales serían necesarias y a qué objetivos de mediano plazo se podría aspirar?

De manera sintética, el sector de CyT, o al menos una parte importante del mismo, debería ser capaz de:

1. Asumir que la debilidad crónica en las políticas de CyT y su desconexión de la realidad socioeconómica local son un producto de la dependencia cultural y económica, consecuencia del lugar asignado a la Argentina en el orden capitalista por las economías centrales en coordinación con los organismos de gobernanza global, como FMI, Banco Mundial, OMC, OCDE. Y que esta posición subordinada es reforzada por los intereses de las fracciones concentradas locales -financieras, extractivas y de baja elaboración de materias primas-, que se alinean y benefician con este campo de fuerzas.

2. Superar auto-representaciones y mecanismos de reproducción que el sector de CyT ha generado a lo largo de su evolución, que son en buena medida una consecuencia de la emulación (simplificada y descontextualizada) de los sectores de CyT de los países centrales. En especial, el subsector de la ciencia académica debe superar el mito liberal fundado en una concepción meritocrática individualista que reclama libertad de investigación y libre competencia en "el mercado de las ideas", mientras que alguna "mano invisible" se encargaría de conectar el conocimiento así producido con las necesidades de la sociedad y el país².

3. Consensuar un cambio de paradigma en la concepción del rol social y económico de la CyT que se oriente a movilizar masivamente las capacidades de investigación y desarrollo (I+D) detrás de un proyecto de cambio estructural y, por lo tanto, a enraizar las agendas de producción de conocimiento en las políticas de industria, salud, energía, defensa, transporte, agro, desarrollo social e infraestructura ${ }^{3}$.

4. Impulsar instancias de organización que vehiculicen los procesos de construcción de consensos alrededor de los objetivos explicitados en la política de CyT, de convergencia con sectores aliados, como gremios industriales, asociaciones empresarias nacionales -con foco especial en PyMEs-, movimientos

\footnotetext{
${ }^{1}$ La idea de "mercado de las ideas" en ciencia puede verse en Polanyi (1962).

${ }^{2}$ Aclaremos que no se trata de atribuir un "pecado original" al sector de CyT, sino que hablamos de consecuencias de procesos históricos en entornos políticos, económicos e institucionales de alta inestabilidad.

${ }^{3}$ Para una discusión de la noción de cambio estructural en Argentina, puede verse el libro de la CEPAL: Abeles et al. (2017).
} 
sociales y sectores de la producción popular -sistemáticamente relegados (invisibilizados) en las iniciativas de producción de conocimiento-4 ${ }^{4}$ y con los sectores de CyT de países de la región. Estas instancias de organización para alcanzar consensos y convergencias son la condición de posibilidad para que el sector logre transformarse en actor político relevante.

Ahora bien, ¿existen las condiciones para esta transformación cultural e ideológica? Un porcentaje importante del sector de CyT apoyó en las elecciones al gobierno actual. Sin embargo, incumpliendo promesas de campaña, desde los primeros meses el macrismo explicitó un proyecto de país con creciente desigualdad, desindustrialización, financierización, endeudamiento y devastación de las actividades de I+D con acelerada precarización del mundo del trabajo. Como respuesta, los/as trabajadoras/es del sector -científicas/ os, tecnólogos/as, ingenieras/os, técnicas/os, administrativos/as--, por encima de sus diferencias, fueron convergiendo en la resistencia a estas políticas. Desde mediados de 2018 no se escuchan voces que defiendan públicamente la "política" de CyT del gobierno.

Esta evolución pone en evidencia una moraleja que, de paso, responde la pregunta: ¿por qué el sector de CyT debe transformarse en actor político a través de consensos internos y convergencias intersectoriales a pesar de su heterogeneidad ideológica y política? La respuesta evidente hoy es: porque la historia argentina y latinoamericana demuestra que las políticas de CyT de los países de la región no logran estabilizarse como políticas de Estado. Dicho de otra manera, hace falta que el propio sector, como actor político, sea un catalizador de la estabilidad de metas estratégicas de I+D como políticas de Estado. Esta puede ser su contribución a la consolidación de la democracia argentina y a la alternancia democrática, problema crucial que discutiremos enseguida, además de ser condición de posibilidad de un proyecto de cambio estructural.

En todo caso, se trata de transformaciones que no pueden ocurrir de forma espontánea. Estas transformaciones no pueden concebirse sin una comprensión profunda de las debilidades estructurales del sector de CyT argentino y sin avanzar en la creación de instancias institucionales que canalicen los consensos y convergencias y materialicen la emergencia del sector de CyT como actor político relevante de un proyecto de cambio estructural. A modo de ejemplo, pueden rescatarse algunos rasgos de la Sociedade Brasileira para o Progresso da Ciência (SBPC) y algunos segmentos de su trayectoria, que reúne a 15-20 mil investigadoras/es anualmente en distintas ciudades de Brasil para consensuar temas de agenda de I+D que el sector de CyT brasileño acuerda como prioritarios ${ }^{5}$. Como contrapunto, preguntémonos a quién representa la Asociación Argentina para el Progreso de las Ciencias (AAPC) o en qué otra instancia institucional está representada la entidad "comunidad de CyT argentina". ¿Por qué la matriz institucional "Asociación para el Progreso", que vehiculizó en muchos países la dimensión política de las comunidades nacionales de CyT, en la Argentina fracasa? Pero entonces, ¿existe el sector de CyT argentino -o una comunidad de CyT argentina- más allá de un conglomerado desconectado de instituciones, gremios, asociaciones profesionales, asociaciones disciplinarias y grupos?

Es decir, si fuera posible un proceso de consensos y convergencias que apunte a redimensionar la CyT como

\footnotetext{
${ }^{4}$ En este punto, en el número anterior de Ciencia, Tecnología y Política, Dagnino (2018: 21) proponía pautas para ir hacia "una tecnociencia capaz de viabilizar la Economía Solidaria mediante el desarrollo de Tecnología Social" en un marco analítico-conceptual coherente con un "proyecto político popular y de izquierda".

${ }^{5}$ Una excelente historia de la SBPC puede verse en Fernandes (2000).
} 
política de Estado, acompañado de un cambio de paradigma en la concepción del rol social y económico del conocimiento, estas transformaciones no pueden ocurrir en el vacío, sino que deben ser concebidas como un proceso integrado a la reconstrucción de la trama social, la recuperación de las capacidades del Estado -entre ellas, la definición de sectores estratégicos y de un proyecto de país industrial con creciente equidad y ampliación de derechos- y la redefinición del lugar de la Argentina en el escenario regional y global.

En síntesis, de este proceso de transformación sistémica deben emerger, además de un sector de CyT como actor político consolidado en instancias organizacionales e institucionales, las alianzas sociales y políticas estratégicas -con gremios, grupos empresarios, movimientos sociales, sectores de CyT de países de la región- y, finalmente, también debe emerger de este proceso el mapa del conocimiento necesario en el que debe enfocarse una política de CyT -las agendas de I+D- capaz de impulsar estrategias eficaces para superar, en el caso argentino, lo hecho hasta 2015 y retomar procesos de aprendizaje, escalamiento organizacional y tecnológico, y transformación cultural que acompañen un proyecto de cambio estructural de escala nacional. Seguramente las ciencias sociales y los doctorados en ingeniería se perfilarían como las necesidades inmediatas más notorias.

\section{Necesidad de una lectura histórica}

Con el macrismo en el poder, la "lógica pendular" de la historia argentina cobró plena actualidad y sentido. El ascenso del yrigoyenismo, su clausura por un gobierno de facto y el "fraude patriótico" (1916-1943); el primer peronismo, el impulso de la industria y los derechos laborales, y la reacción "des-peronizadora" y refundacional del ciclo de dictaduras y semi-democracias (1943-1976); el cuarto de siglo de neoliberalismo dependiente que se inició con una dictadura con pretensiones refundacionales y un genocidio, que puso fin al primer ciclo de cuatro décadas de industrialización y llevó al país al abismo de una crisis terminal (1976-2001); el retorno de un ciclo de gobiernos industrializadores que recuperan metas de redistribución y justicia social seguidos de otro período de neoliberalismo dependiente en curso que se propone "refundar la república" y "des-kirchnerizar" el Estado y la política (2003-2019). Aclaremos que expresiones como "des-peronizar" y "des-kichnerizar", en los hechos, significaron la destrucción del Estado y, con especial virulencia, de las capacidades tecnológicas.

Este siglo de historia revela un patrón de rupturas económico-políticas que lograron bloquear la construcción de un sendero democrático capaz de sostener un proceso de alternancia democrática con políticas de Estado consensuadas por la sociedad, orientadas al desarrollo social y económico centrado en la industrialización con capacidades tecnológicas y científicas que lo hicieran viable en el largo plazo. En este laberinto, el complejo de instituciones del sector nunca logró consolidarse en un sistema de CyT. La clausura periódica de los procesos de expansión y diversificación organizacional e institucional revierten la acumulación de aprendizajes colectivos en gestión de la tecnología, en modalidades de coordinación al interior del Estado y de vinculación público-privada, de incentivo y/o disciplinamiento de sectores y subsectores, etc. Una gran variedad de producción en ciencias sociales no deja el mínimo resquicio de duda respecto del liderazgo activo, protagónico y creativo de los Estados en los procesos exitosos de 
desarrollo social y económico

Por otro lado, como contracara del mismo patrón de rupturas, los sectores empresariales nacionales con capacidad de inversión en I+D consolidan y perfeccionan conductas predatorias y especulativas. A modo de ejemplo, una de las mayores especialistas en políticas industriales y tecnológicas en países de industrialización tardía, Alice Amsden, explica que el problema de la competitividad de las empresas argentinas durante los años noventa -que reclamaron e impusieron un régimen de flexibilización laboral para, supuestamente, mejorar la competitividad- eran los empresarios y no los trabajadores. Amsden explica que las empresas argentinas "no tenían profesionalizadas sus capacidades de gerenciamiento y pocas contaban con planificaciones o cadenas de mando bien definidas". Y agrega: "Las inversiones en I+D fueron insignificantes, por lo que los trabajadores calificados no eran empleados en emprendimientos de alta tecnología. Incluso las plantas con una escala eficiente mínima eran pocas y desconectadas entre sí. [...] el centro de gravedad de la economía argentina volvió a ser el campo, que se caracterizó por una de las distribuciones de ingresos más desiguales del mundo" (Amsden, 2001: 63).

La metáfora del "péndulo argentino", concebida a comienzos de los ochenta por el economista Marcelo Diamand (1983), captura un rasgo esencial del estancamiento dinámico que caracteriza la evolución económica de la Argentina: la "oscilación" no ocurre entre dos proyectos de país que puedan alternar en contexto democrático -aun cuando se vote-, dado que se trata de proyectos que se excluyen y que, por lo tanto, excluyen la posibilidad de políticas de Estado. En un extremo del péndulo hay un proyecto de país que, con límites y contradicciones, supone industrialización, emergencia de sectores estratégicos, políticas sociales y tendencia a la equidad. Cuando el péndulo se mueve hacia el otro extremo, el proyecto de país alternativo siempre impulsa discursos y acciones refundacionales -y, por lo tanto, autocráticos o francamente autoritarios- e inicia procesos de disolución de la soberanía y de reversión de políticas y acciones orientadas hacia la independencia económica y la autonomía tecnológica para favorecer los intereses heterogéneos de fracciones concentradas.

En el primer caso, las gestiones de gobierno deben enfrentar bloqueos, embargos, boicots, operaciones de desestabilización financieras y/o mediáticas y/o judiciales. En el segundo caso, los gobiernos se alían con los intereses que le asignan a la Argentina un lugar en una semiperiferia dependiente y que maximizan ganancias a través de la primarización y la extranjerización de la economía y, desde el último cuarto del siglo XX, a través de la financierización y la gestión del endeudamiento inducido.

Hacia un extremo de la oscilación, el péndulo incrementa la densidad institucional y las capacidades estatales, la matriz productiva se complejiza y surgen agendas dinámicas de investigación y desarrollo $(I+D)$. Cuando la oscilación ocurre hacia el extremo opuesto, se erosiona el Estado, el país se desinstitucionaliza, las tramas productivas se disgregan, la nación se degrada en territorio de extracción y desposesión, la CyT se vuelven innecesarias y tienden a la disolución. Hacia un lado del péndulo se intenta reiniciar procesos colectivos de aprendizaje, expansión, diferenciación y acumulación de capacidades, y escalamiento tecnológico; hacia el otro extremo se inician procesos de disipación de capa-

\footnotetext{
${ }^{6}$ Ver, por ejemplo, una puesta al día de la noción de "Estado desarrollista" en Fine et al (2013) , Thurbon, E. y Weiss, L. (2016)
} 
cidades y desaprendizajes, mientras aumentan en paralelo los flujos de tecnología importada llave en mano, se aceleran los procesos de fuga de cerebros, de retiros voluntarios (incluido el personal más calificado), de desfinanciamiento de institutos y universidades, y desaparecen los vínculos siempre incipientes de las actividades de I+D con el sector productivo. Es este último lado del péndulo el que arrasa con la alternancia democrática o, en todo caso, hace posible solo una alternancia ficticia que niega las políticas de Estado.

En este laberinto bipolar, la tecnología aparece como campo de batalla, blanco de ataque y talón de Aquiles que hace funcionar la trampa geopolítica y geoeconómica que mantiene a la Argentina estancada en una semiperiferia dependiente?. El Estado argentino invirtió en petroquímica desde la creación de YPF en 1922 y de sus laboratorios de Florencio Varela para I+D en 1940; en aeronáutica desde la creación de la Fábrica Militar de Aviones en 1927; en producción pública de medicamentos desde la creación de la Empresa Medicinal del Estado (EMESTA) en 1946; en el sector nuclear desde la creación de la CNEA en 1950; en el sector automotriz con la creación de Industrias Aeronáuticas y Mecánicas del Estado (IAME) desde 1952; en el sector naval desde la creación de Astilleros Río Santiago en 1953; en tecnología de cohetes con la creación de la Comisión Nacional de Investigaciones Espaciales (CNIE) en 1960. También invirtió con paciencia en electrónica y computación: la Facultad de Ingeniería de la UBA fabricó la computadora CEFIBA en 1960, se creó el Instituto de Cálculo de la FCEyN de la UBA en 1963, el INTI creó su grupo de semiconductores por esos días y la empresa FATE abrió su División Electrónica a fines de los años sesenta y desarrolló varios prototipos de computadora a comienzos de los años setenta. Cuando la Argentina canceló el desarrollo de cohetes en 1991, se reorientó a invertir en aprender tecnología satelital. Y apuntalando estos sectores estratégicos, además creó el Instituto Malbrán (1916), universidades públicas, incluidas la Universidad Tecnológica Nacional (1949), el Instituto Balseiro (1955), el Instituto Antártico (1951), CITEFA (1953, hoy CITEDEF), el INTA (1956), el INTI (1957) y el CONICET (1958). A la lista debemos sumar algunas empresas públicas y mixtas y algunos hitos de inversión privada, como Siam, la empresa metalmecánica más importante de América Latina a comienzos de los años sesenta, o el Centro de Investigaciones Industriales del grupo Techint, por ejemplo.

Si la Argentina produjera exportaciones en la mitad de estos sectores o, incluso, en su tercera parte, hoy podría ser un país desarrollado. Pero la Argentina importa todo el equipamiento para la extracción del gas y petróleo de Vaca Muerta -incluidos los servicios de mantenimiento y software-, y la casi totalidad de los bienes de capital para su frágil industria. No exporta aviones, no exporta medicamentos desarrollados en el país, ni reactores nucleares de potencia (los que producen electricidad), tampoco exporta barcos, ni automóviles con porcentaje razonable de componentes nacionales, ni tiene su propio lanzador satelital a pesar de lanzar cohetes desde 1960, ni exporta computadoras o, en general, electrónica de consumo producida por firmas nacionales. Como premio consuelo, sí logró fabricar y exportar reactores nucleares de investigación y pudo fabricar y poner en órbita dos satélites geoestacionarios, con proyección de exportación a países de la región, aunque el gobierno actual clausuró el Arsat 3 y dilapidó toda la inversión pública en este nuevo sector. También hay en el país algunas firmas

\footnotetext{
7 Ver, por ejemplo: Hurtado (2015); Hurtado y Souza (2018).
} 
nacionales de biotecnología, pero sus innovaciones "no mueven la aguja".

De esta forma, la historia del estancamiento dinámico de la Argentina en el laberinto de una semiperiferia dependiente se puede comprender a través de su historia tecnológica y científica. ¿Qué obstáculos se oponen al desarrollo autónomo de tecnologías estratégicas y de agendas de conocimiento útil? El obstáculo determinante y constitutivo es geopolítico: el lugar asignado a América Latina en el orden capitalista mundial explica los "fracasos" tecnológicos y científicos que se manifiestan en la falta de capacidades organizacionales e institucionales para la gestión de tecnologías estratégicas -o "tecnologías tecnologizantes", como las Ilamaba Jorge Sabato (1973), estratega del plan nuclear argentino-y de conocimiento útil para el desarrollo social y la complejización de la trama productiva. El complemento de esta debilidad estructural son culturas empresariales proclives a la aversión al riesgo, al cortoplacismo y a las conductas rentísticas anti-emprendedoras, y culturas científicas que reclaman financiamiento público y son proclives a insertarse en los circuitos de producción de conocimiento útil de las economías centrales.

\section{La última oscilación del péndulo}

Un ejemplo didáctico de la ausencia de alternancia democrática -o de alternancia ficticia-, a pesar de la vigencia del voto, es la llegada de la alianza Cambiemos al gobierno con una diferencia de 2\% de votos en ballotage. Esta gestión de gobierno hasta la fecha no solo no cumplió con ninguna promesa de campaña, sino que además, clausurando políticas públicas, impulsa un proyecto refundacional de desindustrialización, extractivismo, financierización, endeudamiento, extranjerización y fuga de capitales y, en este contexto, avanza en la devastación del sector de CyT. Para quienes lograron permanecer más o menos inmunizados a las operaciones mediáticas, culturales y políticas de la tríada del poder fáctico -periodismo de guerra oligopólico, operaciones de inteligencia y persecuciones judiciales- resulta claro que la "lógica" histórica del péndulo argentino se manifiesta en el presente con especial virulencia como consecuencia de la deriva del capitalismo global, marcada por la crisis de 2008, la financierización descontrolada de la economía global y el ascenso de China, que se manifiesta en América Latina en la reacción intervencionista de EEUU y el protagonismo de las corporaciones financieras a través de la cooptación del Estado argentino. ¿Cómo pensar la evolución del sector de CyT en este escenario?

Como parte del proceso de recuperación de la crisis terminal de 2001, cuando se creó el MINCyT a fines de 2007 y, en paralelo, el gobierno promovió una resignificación del lugar social y económico de la producción de conocimiento, se debió enfrentar una herencia inequívoca de dependencia cultural: no existía (no existe tampoco hoy) una lectura política densa del lugar de la CyT en la batalla por el desarrollo social y económico; dominaban auto-representaciones dentro del sector de CyT argentino fundados en imaginarios funcionales a su auto-reproducción en contextos adversos; incluso en la retórica de muchos funcionarios del sector prevalecían concepciones que fueron impuestas durante los años noventa y que lograron traspasar la crisis de 2001. Sin embargo, las políticas de CyT durante 2003-2015 dejaron enormes aprendizajes, transformaron de manera irreversible el escenario material 
y avanzaron en lo que podríamos llamar la "batalla cultural" por una inserción de las agendas de producción de conocimiento en un proyecto de país.

A contramano del lugar asignado a la Argentina en el orden capitalista mundial, durante el período 2003-2015 el gobierno logró avanzar en la transformación del imaginario de una buena parte del sector de СуТ -con especial influencia sobre las generaciones más jóvenes- y también logró incidir sobre la percepción social de la CyT a través de hitos como Tecnópolis, el canal Encuentro o el Programa Raíces. En diciembre de 2015, este proceso quedó en la mitad del río y no alcanzó un umbral que hiciera posible abordar, con fines de transformación estructural, cuestiones de enorme complejidad e importancia, que van, por ejemplo, desde la consolidación de una industria de bienes de capital o una política energética con componentes robustos de política industrial y de I+D hasta el avance de planes de sustentabilidad ambiental de las actividades extractivas, como la megaminería o el uso de agroquímicos. Llegar a las PyMEs o a la economía social y solidaria podrían ser otros ejemplos. En términos generales, las deficiencias de la política industrial y la tendencia al encapsulamiento del MINCyT pusieron límites a la eficacia de las políticas.

A la pregunta "¿por qué el proceso quedó en la mitad del río?" se podría contraponer otro interrogante: ¿existían en el Estado argentino capacidades suficientes y contaba el gobierno con el poder político necesario para enfrentar a los poderes fácticos? Dicho de otra manera, las condiciones de posibilidad para avanzar sobre cuestiones como extractivismo, megaminería, o uso intensivo de agroquímicos, o para consolidar políticas industriales y de CyT orientadas al cambio estructural, son un Estado inteligente y eficaz -que supone procesos acelerados de acumulación de capacidades técnicas y burocráticas- y un gobierno con el poder político necesario para enfrentar y neutralizar la influencia de los poderes fácticos locales y trasnacionales potenciados desde la arena internacional por "reglas de juego" adversas. Este escenario supone la necesidad de una creciente coordinación del sector de CyT con la sociedad civil, gremios, movimientos sociales y sectores empresarios nacionales, además de alianzas estratégicas con las Fuerzas Armadas -como ocurrió, por ejemplo, con la política de radarización de los aeropuertos ${ }^{8}$ - y el avance en los procesos de integración regional que logren traspasar el nivel de los acuerdos comerciales.

Frente al horizonte electoral en octubre de 2019, la herencia de una Argentina híper-endeudada, donde no queda otro camino, como explica Enrique Martínez (2019), que "establecer como valor cultural concreto que el manejo de las divisas debe ser regulado como lo sería el agua en el desierto", obliga a preguntar: ¿cómo podría movilizar el sector de CyT sus capacidades para ser un actor protagónico en el rescate de la Argentina del infierno de financierización, extranjerización y endeudamiento que nos deja el proceso de saqueo que hoy impulsa el actual gobierno? Responder a la emergencia social y productiva debería ser el primer objetivo del sector de CyT. Esta prioridad podría ser un buen comienzo para una transformación cultural necesaria.

\footnotetext{
${ }^{8}$ En este mismo número de la revista hay un artículo de Juan Martín Quiroga que trata este tema.
} 
| Ciencia, Tecnología y Política | Año 2 | N² | Enero-Junio 2019 | ISSN 2618-3188 | www.revistas.unlp.edu.ar/CTyP |

\section{Bibliografía}

Abeles, M., Cimoli, M. y Lavarello, P. (eds.). 2017. Manufactura y cambio estructural: aportes para pensar la política industrial en la Argentina. Santiago: Comisión Económica para América Latina y el Caribe (CEPAL).

Amsden, A. 1989. Asia's Next Giant. New York: Oxford University Press.

Dagnino, Renato. 2018. "Elementos para una Política Cognitiva popular y soberana", Ciencia, Tecnología y Política, núm. 1, pp. 16-21.

Diamand, M. 1983. El péndulo argentino: ¿hasta cuándo? (folleto). Buenos Aires: Centro de Estudios de la Realidad Argentina.

Dussel, E. 2016. Filosofías del Sur. Descolonización y transmodernidad. México, DF: Akal.

Fernandes, A.M. 2000. A construção da ciência no Brasil e a SBPC. Brasilia: Editora UnB.

Fine, B., Saraswati, J. y Tavasci, D. 2013. Beyond the Developmental State. Industrial Policy into the Twenty-First Century. Londres: Pluto Press.

Hurtado, D. 2015. "Semi-periphery and capital-intensive advanced technologies: The construction of Argentina as a nuclear proliferation country", Journal of Science Communication (Trieste: SISSA), vol. 14, núm. 2, A05. En: http://jcom.sissa.it/archive/14/02/JCOM_1402_2015_A05

Hurtado, D. y Souza, P. 2018. "Geoeconomic Uses of Global Warming: The "Green" Technological Revolution and the Role of the Semi-Periphery", Journal of World-System Review, vol. 24, núm. 1, pp. 123-150.

Martínez, E. 2019. “¿Hay vida después del FMI?”, Agencia Paco Urondo, 18 de febrero. En: http://www. agenciapacourondo.com.ar/opinion/hay-vida-despues-del-fmi-por-enrique-martinez

Polanyi, M. 1962. "The Republic of Science: Its Political and Economic Theory", Minerva, vol.1, pp. 5473.

Sabato, J. 1973. "El comercio de tecnología", Programa de transferencia. Bariloche: Fundación Bariloche.

Thurbon, E. y Weiss, L. 2016. “The developmental state in the late twentieth century”, pp. 637-650. En: Reinert, E., Ghosh, J. y Kattel, R. (eds.), Handbook of Alternative Theories of Economic Development. Cheltenham, UK: Edward Elgar Publishing. 